\title{
Conocimientos sobre Identidad Sexual de Profesores y Profesoras: ¿Barreras o Facilitadores de Construcción Identitaria?
}

\section{Teachers Knowledges about Sexual Identity: ¿Barriers or Facilitators of Identity Construction?}

\author{
Cristina Julio Maturana ${ }^{1 *}$ \\ Anna Kaeuffer ${ }^{2}$ \\ Christopher Riquelme Salinas ${ }^{3}$ \\ María Paz Silva Erices ${ }^{3}$ \\ María Roswitha Osorio Hodges ${ }^{4}$ \\ Natalie Torres Estay ${ }^{5}$
}

${ }^{1}$ Universidad de Playa Ancha de Ciencias de la Educación ${ }^{2}$ Colegio Alianza Alemana ${ }^{3}$ Colegio

Winterhill ${ }^{4}$ Centro de Rehabilitación Cruz del Sur ${ }^{5}$ Colegio Sagrada Familia de Nazareth

Chile ha comenzado a reconocer a la comunidad LGBTI (Lesbianas, Gay, Bisexual, Transexual e Intersexual). Este reconocimiento permite visibilizar sus necesidades sociopolíticas y exige asumirlas con nuevas legislaciones y políticas, entre ellas las de Educación. Este artículo expone la reflexión que emana de los resultados de un estudio exploratorio de tipo cualitativo cuyo objetivo fue develar los conocimientos declarativos, procedimentales y actitudinales sobre identidad sexual, que tienen Educadores de Párvulos y profesores de primero y segundo año de Educación Básica de escuelas municipales. Los hallazgos permiten identificar conocimientos basados en el paradigma socio-constructivista y también en el esencialista y, discutir cómo en los espacios escolares los profesores y las profesoras actúan como facilitadores u obstaculizadores de la construcción de la identidad sexual de niños y niñas, dependiendo de sus conocimientos sobre la materia y el paradigma que los sustente, y cómo los cambios políticos impactan en estos.

Descriptores: Identidad sexual, Inclusión, Conocimientos, Profesoras/profesores, Paradigma socio-constructivista.

Chile has begun to recognize the LGBTI (Lesbian, Gay, Bisexual, Transgender and Intersex) community. This recognition allows make their sociopolitical needs and demands visible and thus to include them within new legislation and policies, including in education. This article presents the reflection emanating from the results of an exploratory qualitative study whose objective was to reveal the declarative, procedural and attitudinal knowledge about sexual identity that teachers from early childhood education and first and second year of primary-level education of three municipal schools have. The findings allow for the identification of knowledge based on a socio-constructivist paradigm and also on the essentialist paradigm and discuss how teachers in school act as facilitators for or hinder the construction of sexual identity of children, depending on their knowledge of the subject and their paradigm, and how policy changes impact these areas.

Keywords: Sexual identity, Inclusion, Knowledge, Teachers, Social constructivist paradigm. 


\section{Introducción}

El presente artículo da cuenta de los resultados de un estudio exploratorio realizado en el año 2013, cuyo problema de investigación emergió de dos circunstancias. Primero, del interés por conocer cómo comprenden la Identidad Sexual los docentes que forman niños y niñas a temprana edad y su incidencia en la construcción temprana de la misma. En segundo lugar, de los escasos estudios realizados entonces, sobre la construcción de la identidad sexual en la infancia y la influencia de los profesores y profesoras en dicho proceso. No obstante, desde ese mismo año se han generado acercamientos a la temática por medio de políticas de reconocimiento a nivel nacional que han visibilizado necesidades sociopolíticas de la comunidad LGBTI (Lesbianas, Gay, Bisexual, Transexual e Intersexual), lo que se ha traducido en leyes tales como la Ley de No Discriminación $\mathrm{N}^{\circ}$ 20.609, la Ley de Acuerdo de Unión Civil; y en educación la Ley de Inclusión y la Política de Convivencia Escolar, las cuales -considerando un enfoque de género, derechos e inclusión- resguardan el derecho a la educación para todos y todas, así como también la denuncia de cualquier tipo de discriminación en el espacio escolar. Es por esto que, aunque el estudio se realizó el 2013, se ha actualizado su discusión profundizando en la relación entre los conocimientos del profesorado, los cambios legislativos actuales y la posibilidad que tienen los niños y niñas de construir su identidad sexual en la escuela.

En el contexto planteado, el estudio realizado se propuso develar los conocimientos declarativos, procedimentales y actitudinales sobre el concepto de identidad sexual que manejaban entonces Educadoras/es de Párvulos y profesores de primer y segundo año de Educación Básica de tres escuelas municipales, con el propósito de conocer la influencia que tienen en la construcción de la identidad sexual de sus estudiantes. Para dar cuenta de ello, el artículo se organiza en tres grandes apartados. Primero una aproximación teórica que sustenta el estudio; segundo, se presenta el método de estudio desarrollado; en tercer lugar, se exponen los resultados a modo de hallazgos y se culmina con una discusión sobre el objeto de estudio, el problema visualizado y los resultados obtenidos.

\section{Revisión de la literatura}

El presente apartado tiene como objetivo sustentar el estudio, sus resultados y discusión. En él se profundiza en los tipos de conocimientos relacionados al concepto de identidad e identidad sexual; cómo estos conocimientos, en el profesorado, se pueden presentar como facilitadores u obstaculizadores del proceso de construcción identitaria en el proceso formativo de niños y niñas; finalizando con aquellos aspectos sociopolíticos que instalan marcos legales nacionales que inciden en políticas públicas de educación en Chile.

\subsection{Concepción de Conocimiento asumido}

$\mathrm{El}$ estudio asume que los conocimientos constituyen un conjunto de saberes culturales, sociales, políticos, económicos, científicos y tecnológicos, que conforman las distintas áreas disciplinares y se consideran esenciales para la formación del individuo (Odreman, 1996). Existen diferentes definiciones de los saberes, una de ellas Delors y colaboradores (1996) plantea el aprender a conocer, aprender a hacer, aprender a ser y aprender a convivir, que son explicados a continuación.

De acuerdo a Delors y sus colaboradores (1996), el conocimiento declarativo es entendido como el aprender a conocer, en donde cada persona comprende, aprehende y descubre 
información del mundo que lo rodea. El conocimiento procedimental lo define como el aprender a hacer, el cual "capacita al individuo para hacer frente a gran número de situaciones y a trabajar en equipo" (p. 109). Por último, el conocimiento actitudinal se relaciona con el saber ser, el cual consiste en el cumplimiento de normas, formación de valores y actitudes que se expresan a través de preferencias y el aprender a convivir. Es importante destacar que tanto el conocimiento declarativo, procedimental como el actitudinal, tienen el mismo grado de importancia en la práctica educativa por lo que deberían abordarse de manera integrada (Woolfolk, 2006).

\subsection{Identidad sexual (IS): Un nuevo conocimiento para profesores y profesoras}

El estudio asume, al profesor o profesora como un ser que comprende y aprehende la realidad sociocultural en la cual está situado, entendiendo la política que erige a su época (Tezanos, 2007). Que posee la habilidad de facilitar en los niños y niñas la construcción de conocimientos (declarativos, procedimentales y actitudinales), así como también debe responder a diversas exigencias laborales encaminadas a formar personas, establecer vinculaciones con los distintos saberes, disciplinarios y técnicos especializados, los que confluyen en su saber propio, y asumir la responsabilidad de construir relaciones entre los distintos actores comprometidos en la tarea común de formar personas (Prieto, 2004). Dadas estas características es posible afirmar con las autoras que ser profesor o profesora es un proceso de construcción identitaria que se va configurando de manera permanente en la interacción con la realidad social, económica, política, histórica y cultural en la que se desarrolla y ejerce como persona-profesional. Por ende, se construye y deconstruye la identidad profesional, y junto a ella se modifican aquellos conocimientos de su saber pedagógico. En este sentido, la identidad sexual se configura como otros de los conocimientos que deberían formar parte del conocimiento y saber pedagógico de un profesor o profesora que ejerce en tiempos actuales.

\subsection{Construcción identitaria en la niñez}

El estudio asume dos miradas acerca de la identidad. Por una parte, asume con Erickson (1963, en Robles, 2008) que la identidad (el yo) de los seres humanos se desarrolla sobre la base de su interacción con su ambiente. En este sentido el estudio asume que la construcción identitaria desde un paradigma sociocultural, debido a que las personas son seres activos y sociales que buscan adaptarse al ambiente en que viven junto a otros. Por otra parte, asume que la identidad es el resultado de la construcción, co-construcción y reconstrucción llevados a cabo por la niña y el niño mediante las interacciones con sus padres y madres, maestros y maestras, compañeros y compañeras, como también, por la influencia recibida a través de los medios de comunicación e información. Tal construcción identitaria se caracteriza por una creciente toma de conciencia de los rasgos distintivos tales como el sexo, el género, la pertenencia a un grupo étnico, la edad y la condición de la comunidad con la cual el niño y la niña están en estrecho contacto (Brooker y Woodhead, 2008).

Por lo expuesto, los niños y las niñas construyen su identidad personal, adquieren aptitudes, conocimientos y conductas valoradas culturalmente, no sólo en la familia sino también en la educación formal a través de padres, madres, cuidadoras o cuidadores, profesores y profesoras, las escuelas y/o su grupo de pares. Ciertamente, el aprendizaje temprano incide en cómo el niño o la niña ve su propio modo de ser, cómo se relacionan con las demás personas y con el resto del mundo (Brooker y Woodhead, 2008), es por ello 
que el estudio pone énfasis en los procesos de socialización y construcción de la identidad en la infancia.

\subsection{Concepto de Identidad Sexual (IS) y su construcción en la niñez}

Como ya fue indicado, el desarrollo de la identidad se encuentra sostenido frente al dinamismo de las interacciones que genera cada persona e involucra la multidimensionalidad del ser, en donde se sitúan desde los cambios físicos y biológicos, hasta el sentir, los cuales también se articulan para configurar la Identidad Sexual. El estudio comprende la Identidad Sexual como la "autoasignación, aceptación o adjudicación consciente, subconsciente o inconsciente (explícita o implícita a la percepción de un yo) de la pertenencia a un estado de psiquismo sexual" (Valenzuela, 1993, en Conejero y Almonte, 2009, p. 202), lo cual implica que "el sujeto asume una denominación reconocida socialmente, que incluye sentimientos, atracciones y conductas sexuales y, es el resultado de la interacción de aspectos biológicos, familiares, sociales, históricos y culturales, los cuales hacen que la identidad sexual se plantee como un estado que puede cambiar" (Almonte, 2007; Shutt-Aine y Maddaleno, 2003 en Conejero y Almonte, 2009). Sobre la base de ésta definición se entiende que la identidad sexual abarca la identidad de sexo, identidad de género y la orientación sexual, (Conejero y Almonte, 2009).

En este sentido para este estudio es relevante conocer los aspectos que constituyen la sexualidad, que desde el punto de vista de Bardi, Leyton, Martínez y Gonzáles (2005), son los siguientes:

- Sexo biológico: Tiene relación con los caracteres morfofuncionales y es la base de la asignación del sexo al recién nacido.

- Identidad de género: Es entendida como la convicción íntima, el sentimiento básico internalizado y la experiencia psicológica interna de una persona, de sentirse a sí mismo/a, como mujer u hombre.

- Rol de género: Refiere al comportamiento definido como masculino o femenino en diferentes épocas y en una cultura determinada.

- Orientación sexual: Es la dirección de los intereses eróticos y afectivos que tiene un individuo por otro, ya sea heterosexual, homosexual o bisexual.

Considerando los conceptos señalados, Polaino-Lorente (2007) explica que sexo y género pueden comunicarse entre sí o, por el contrario, pueden disociarse y romper sus relaciones. Así también, el fenómeno de adquisición de la identidad sexual, según Becerra y Melo (2006) se inicia desde etapas muy tempranas en la vida, siendo éste un proceso dinámico y didáctico, que comienza en las primeras etapas del autoconocimiento por exploración, y posteriormente, mediante una interrelación entre la persona con el medio social, y la confrontación con las y los demás.

Así también la existencia de teorías enmarcadas en un paradigma antiesencialista deconstructivo, como la Teoría Queer, se instalan como una propuesta que interpelan y cuestiona las categorías identitarias planteadas por la mirada hegemónica sobre "la concepción de sujeto/a universalista que sustenta la política liberal actual, como los procesos de esencialización, naturalización e identificación de las teorías de la política de la diferencia con relación al sector LGTBIQ" (Duque, 2010, p. 28). En este sentido, Preciado (2002) y Butler (1993 y 2003, en Duque, 2010) nos emplazan a comprender que conceptos tales como el sexo, género, rol de género y orientación sexual, emergen a partir de una 
construcción-producción social, cultural e histórica y que, por lo tanto, pueden ser deconstruidas para la conformación de un nuevo orden simbólico y social, que permita el reconocimiento de la complejidad y diversidad humana.

\subsection{Conocimiento del profesorado: ${ }_{\text {iFacilitadores u obstaculizadores del proceso }}$ de construcción de la identidad sexual (IS)?}

El estudio asume que el proceso de construcción de la identidad profesional y el saber pedagógico, es lento, continuo y está en constante transformación debido a los cambios sociales, culturales y políticos (Prieto, 2004; Romero, 2010) del sistema educativo al que pertenece el profesor o la profesora. En esta constante reconstrucción identitaria tanto de profesores/as como de estudiantes inciden las experiencias y conocimientos previos así como las identidades de cada cual por lo que ambos pueden considerarse agentes obstaculizadores o facilitadores de la construcción identitaria del otro. Este estudio se ocupa del profesorado, la incidencia de sus conocimientos y saberes pedagógicos en la construcción de la IS de sus estudiantes en los primeros años de escolaridad.

En este sentido, Debís, Fuentes y Sparkes (2005) afirman que los profesores y las profesoras son agentes obstaculizadores en el proceso de construcción de identidad sexual, por medio del currículum oculto, refiriendo a que dichos valores, actitudes y formas de interacciones sociales que los alumnos y las alumnas aprehenden durante su proceso de escolarización, condicionan las identidades de los y las mismas, construyendo regímenes de verdad, tomando como punto de partida el género, a través de actividades que separan sus roles y conductas según su sexo. Sin embargo, las profesoras y los profesores como facilitadores pueden transgredir y transformar los espacios delimitados por las escuelas, y trabajar no sólo en el aula, sino que también en talleres extracurriculares, en la sala de profesores, en el patio, etc., de modo que se logre la visibilización de estas temáticas (Hemingway, 2008; Pozo y Monereo, 1999). Asimismo, Prengel (2011) hace hincapié en que los profesores y las profesoras contribuyen en la educación y en la construcción de la identidad sexual de sus estudiantes, puesto que es él o ella quien define las condiciones materiales y culturales de vida, dentro de las cuales el niño o la niña se va desarrollando.

\subsection{Algunos antecedentes sociopolíticos referidos a IS en la educación}

El estudio consideró algunos antecedentes sociopolíticos que permiten sustentar la incidencia de las políticas públicas de educación en el conocimiento del profesorado y sus saberes pedagógicos y cómo estos a su vez facilitan o dificultan la construcción de la IS en niños y niñas a temprana edad. A partir de la década del 60, en Chile se integró la educación sexual a los programas gubernamentales, no obstante, en la Dictadura Militar, Pinochet elimina del currículo escolar cualquier referencia o actividad que no abordará la biología de la reproducción. Después de la dictadura, se integran aspectos de educación sexual y afectiva al currículo nacional. Actualmente, ésta temática está incorporada al Currículo Nacional mediante los objetivos fundamentales, abarcando algunos de los conceptos asumidos en el estudio. Sin embargo, según Valenzuela (1993, en Conejero y Almonte, 2009), con énfasis distintos, ya que, por un lado en Educación Parvularia se desarrolla más el concepto de identidad en sí misma, en Básica se enfatizan las características morfofuncionales.

Respecto al sistema político, en Chile se han incorporado diferentes leyes relacionadas con la inclusión en general y con temáticas vinculadas con la identidad sexual en particular. Estas son: La ley n 20.609/2012, Antidiscriminación; la ley n²0.830/2015, Acuerdo de 
Unión Civil; la ley $n^{\circ}$ 20.845/2015 de Inclusión Escolar que incluye la política de Convivencia Escolar 2015-2018. La ley Antidiscriminación establece que "Toda distinción, exclusión o restricción que carezca de justificación razonable...” (2012, art. 2) puede ser denunciada e incluye explícitamente discriminaciones en relación a "el sexo, la orientación sexual, la identidad de género". La ley de acuerdo de unión civil "es un contrato celebrado entre dos personas que comparten un hogar, con el propósito de regular los efectos jurídicos derivados de su vida afectiva en común, de carácter estable y permanente"(2015, art.1). Este contrato puede establecerse entre personas del mismo sexo o sexos diferentes y regula el estado civil de los convivientes. La ley de inclusión escolar "regula la admisión de los y las estudiantes, elimina el financiamiento compartido y prohíbe el lucro en establecimientos educacionales que reciben aportes del estado" (Ley n ${ }^{\circ}$ 20.845); resguardando el derecho de los y las estudiantes a la educación y abriendo la posibilidad de denunciar discriminaciones de diversos tipos, entre otros las relacionadas con la identidad sexual. De esta ley emana, entre otras cosas, una política de convivencia escolar, la cual pretende -mediante la fiscalización y orientación de los reglamentos de convivencia escolar-eliminar múltiples discriminaciones y apoyar la puesta en práctica de la ley de inclusión. Es por esto que sus ejes centrales, entre otros, son el enfoque de género, el de inclusión y el de derechos (MINEDUC, 2015).

Finalmente, y como ya fue dicho, el estudio explora, sobre la base de las comprensiones indicadas, los conocimientos que un grupo de profesores y profesoras tiene sobre IS y cómo estos inciden en la construcción identitaria de sus estudiantes. A continuación se expone el método utilizado para tal exploración.

\section{Método}

El estudio se sustentó en un diseño exploratorio, de tipo cualitativo e interpretativo (Hernández, Fernández y Baptista, 2003), por lo que los resultados obtenidos, se remiten a la particularidad de cada caso analizado, y dependen del contexto concreto y de las relaciones establecidas entre quien investiga y quien informa (Lincoln y Guba, 1985).

El procedimiento de recolección de información utilizado se sustentó en entrevistas individuales semiestructuradas (Grinell, 1997), realizadas a ocho profesores de Educación Parvularia y Básica, según se expone en la tabla 1. Éstas fueron realizadas en una sola sesión, siendo registradas en audio tal como fueron relatadas por las y los educadores, para luego de transcribirlas, tratar de comprender dichos relatos (Vargas, 2012).

Los informantes claves se seleccionaron de manera directa e intencionada, considerando criterios de: Coherencia con los objetivos, Pertenencia a escuelas municipales y pertenecientes al nivel de Educación Parvularia y primero y segundo de Educación Básica, tal como se presentan en la siguiente tabla. 
Tabla 1. Informantes claves

\begin{tabular}{|c|c|c|c|}
\hline ESCUELA & INFORMANTES & NiVEL EdUCATIVO & ENTREVISTA INDIVIDUAL \\
\hline \multirow{4}{*}{ Escuela 1} & 1 Profesora NT I & & \multirow{4}{*}{$100 \%$} \\
\hline & 1 Profesor NT II & $2 \mathrm{EPA}$ & \\
\hline & 1 Profesora de $1^{\circ}$ Básico & $2 \mathrm{EBA}$ & \\
\hline & 1 Profesora de $2^{\circ}$ Básico & & \\
\hline \multirow{3}{*}{ Escuela 2} & 1 Profesora NT I & & \multirow{3}{*}{$100 \%$} \\
\hline & 1 Profesor NT II & & \\
\hline & 1 Profesora de $2^{\circ}$ Básico & 1 EБA & \\
\hline Escuela 3 & 1 Profesora de $1^{\circ}$ Básico & $1 \mathrm{EBA}$ & $100 \%$ \\
\hline Total & 8 Profesores & $4 \mathrm{EPA}+4 \mathrm{EBA}$ & $100 \%$ \\
\hline
\end{tabular}

Fuente: Elaboración propia.

Para el análisis de los relatos o narraciones, se usa un método mix to (etic-emic). Etic porque antes del análisis y sobre la base de referentes teóricos que sustentan el fenómeno del conocimiento del profesorado, se definieron conceptual y operacionalmente categorías referidas a los tipos de conocimientos (declarativo, procedimental y actitudinal) considerando. Emic porque los indicadores establecidos permitieron configurar categorías emergentes con posterioridad al análisis. Por ello, tal como se expone en la tabla 2 , se define el conocimiento declarativo, como homólogo al conocimiento reflexivo planteado por Tezanos (1983); el conocimiento procedimental al conocimiento productivo dado por la misma autora; y por último, se define el conocimiento actitudinal desde los planteamientos de Doménech (1999), operacionalizando indicadores que permiten identificar los conocimientos en los relatos registrados.

Se realiza un análisis de contenido (Cáceres, 2003) de las narraciones del profesorado. Este análisis se realiza con posterioridad a la transcripción de las entrevistas y a la construcción de un corpus de contenido. El análisis propiamente tal se realiza sobre la base de los indicadores definidos (Cáceres, 2003), estableciendo unidades de análisis (Morris, 1994, en Cáceres, 2003) y codificaciones que permiten las categorizaciones emergentes (Hernández, 1994, en Cáceres, 2003; Osses, Tapia y Ibáñez, 2006) en cada tipo de conocimiento (declarativos, procedimentales y actitudinales) establecido. Finalmente, los contenidos obtenidos en virtud de las unidades definidas permiten la triangulación de los resultados con las convicciones de las y los investigadores/as y el marco referencial; lo que permite obtener y explicitar los hallazgos que a continuación se exponen.

\section{Hallazgos}

Los conocimientos que emergieron del análisis de los relatos de profesores y profesoras se presentan en una tabla de doble entrada que presenta categorías y subcategorías emergentes por tipo de conocimiento. Es así como en la tablas 3 se exponen los conocimientos declarativos que emergieron, en la tabla 4 los actitudinales y en la tabla 5 los procedimentales. En cada tabla, la primera columna corresponde a las categorías que emergieron, mientras que la segunda corresponde a las subcategorías. Haciendo lectura de izquierda a derecha, las categorías se desglosan en las subcategorías correspondientes que posteriormente se desarrollan y se sustentan en datos emic.

Tabla 2. Definición conceptual y operacional de tipos de conocimientos 


\begin{tabular}{|c|c|c|}
\hline $\begin{array}{c}\text { TIPO DE } \\
\text { CONOCIMIENTO }\end{array}$ & $\begin{array}{c}\text { DEFINICIÓN EN EL } \\
\text { ESTUDIO }\end{array}$ & INDICADORES PARA EL ANÁLISIS \\
\hline Declarativos & $\begin{array}{l}\text { Conocimientos basados en } \\
\text { definiciones que } \\
\text { dominan los profesores } \\
\text { y las profesoras de EPA, } \\
\text { y de primer y segundo } \\
\text { año de EBA sobre } \\
\text { identidad sexual, y cómo } \\
\text { ésta se construye en la } \\
\text { infancia }\end{array}$ & $\begin{array}{l}\text { Hacer mención a una o más dimensiones de la } \\
\text { identidad sexual: identidad de género, sexo } \\
\text { y/u orientación sexual. } \\
\text { Acercarse al concepto de identidad sexual } \\
\text { identificándolo como dinámico, } \\
\text { multidimensional e interpersonal. } \\
\text { Nombrar algunos de los factores que influyen } \\
\text { en la construcción de la identidad sexual } \\
\text { como la familia, las escuelas, profesores y } \\
\text { profesoras, y las culturas. }\end{array}$ \\
\hline Procedimentales & $\begin{array}{l}\text { Prácticas pedagógicas que } \\
\text { los profesores y las } \\
\text { profesoras de EPA, y } \\
\text { primer y segundo año de } \\
\text { EBA tienen, en relación } \\
\text { al concepto de identidad } \\
\text { sexual. }\end{array}$ & $\begin{array}{l}\text { Asignación de trabajos en el aula; elección de } \\
\text { juegos y juguetes; el reconocimiento del } \\
\text { sexo, el género y orientación sexual como } \\
\text { elementos constitutivos de la identidad } \\
\text { sexual. } \\
\text { Enseñanza en base a la aceptación y tolerancia } \\
\text { de la diversidad en el aula, incluyendo la } \\
\text { identidad de género; si educan sexualmente, } \\
\text { buscando soluciones mediante la } \\
\text { conversación tanto con la y el estudiante } \\
\text { como con los padres. }\end{array}$ \\
\hline Actitudinales & $\begin{array}{l}\text { Aquellos valores, } \\
\text { actitudes y normas } \\
\text { intrínsecas de las } \\
\text { profesoras y los } \\
\text { profesores que dirigen el } \\
\text { qué hacer docente, en } \\
\text { relación a los procesos } \\
\text { pedagógicos y sus } \\
\text { implicancias en la } \\
\text { construcción de la } \\
\text { identidad sexual en la } \\
\text { infancia }\end{array}$ & $\begin{array}{l}\text { Aceptación, tolerancia y trabajo para la } \\
\text { diversidad de identidades, en un trato } \\
\text { igualitario entre las y los estudiantes sin } \\
\text { importar sus características de sexo, } \\
\text { identidad de género y orientación sexual. } \\
\text { Asimismo, los indicadores que se } \\
\text { desarrollaron en los conocimientos } \\
\text { procedimentales, se presentan como un } \\
\text { reflejo de lo actitudinal, puesto que las } \\
\text { prácticas surgen en tanto a los valores y los } \\
\text { conocimientos que guían el quehacer } \\
\text { docente. }\end{array}$ \\
\hline
\end{tabular}

Fuente: Elaboración propia.

\subsection{Conocimientos declarativos de profesoras y profesores, sobre identidad sexual}

Tal como se presenta en la tabla 3, las categorías que emergieron en relación a los conocimientos declarativos acerca de la identidad sexual, son: A) biológicas, B) de género, C) de orientación sexual, D) multidimensional, E) de construcción y desarrollo permanente y F) de la influencia de la familia.

a) Identidad sexual como un componente biológico

El conocimiento declarativo sobre identidad sexual desde una concepción biológica en este estudio se comprende según Bardi y colaboradores (2005). Un ejemplo de esto es:

Mira la identidad sexual (...) es el reconocimiento con su propio cuerpo y con su identidad como niño o niña, el cuerpo humano, te dicen las partes, las funciones, que lo que hacen (...) el niño diferencia bien los genitales, que sepa que el nombre de los genitales del hombre son testículos, pene, las mujeres, la vulva, la vagina, me entiendes. (P2-EPA, p. 1) 
Tabla 3. Conocimientos declarativos de profesoras y profesores, sobre identidad sexual

\begin{tabular}{ll}
\hline \multicolumn{1}{c}{ CATEGORÍAS EMERGENTES } & \multicolumn{1}{c}{ SUBCATEGORÍAS EMERGENTES } \\
\hline A. Biológico & A.1 Factor genético \\
\hline \multirow{2}{*}{ B. Género } & B.1 Identidad de género \\
& B.2 Roles de género \\
\hline & C.1 Desde una posición heterosexista \\
& C.2 Condicionamiento de la orientación sexual al \\
C. Orientación Sexual & S.2 Como concepto dinámico \\
& C.3 Por exclusión \\
\hline & D.1 Cultura \\
D. Multidimensional & D.2 Emoción y Afectividad \\
& D.3 Multidimensional del ser \\
\hline E. Construcción y Desarrollo & E.1 Experiencia \\
Permanente & E.2 Infancia \\
\hline F. La Familia Influye & F.1 Crianza, Modelo o Referente \\
\hline
\end{tabular}

Fuente: Elaboración propia.

\section{b) Identidad sexual como un componente de género}

Asimismo comprender la identidad sexual refiriéndose a una perspectiva de género es entendida, como descrito anteriormente, según Bardi y colaboradores (2005), lo que se evidencia por ejemplo en la siguiente respuesta: "Yo me imagino que es como una identidad que tú tienes, si eres hombre o eres mujer" (P6- EBA, p. 1-2).

\section{c) Identidad sexual como un componente de orientación sexual}

Un tercer conocimiento declarativo de identidad sexual que tienen los profesores y las profesoras es la orientación sexual, entendida según Bardi y colaboradores (2005). Esto se evidencia que, ante la consulta de uno o una de sus alumnos o alumnas, con respecto a si "algunas personas se casan con personas del mismo sexo", el o la educadora afirma "yo le llegué a explicar, con la ayuda también de la otra tía, que había que sí se daban esos casos, pero que no era lo normal, pero si podían llegar a darse" (P1-EPA, p. 3).

\section{d) Identidad sexual desde una perspectiva multidimensional}

Un cuarto conocimiento declarativo sobre identidad sexual refiere a una concepción multidimensional, entendiendo esta como las diversas maneras que se utilizan para definir un fenómeno, evidenciándose en el planteamiento de un o una profesor o profesora de EBA:

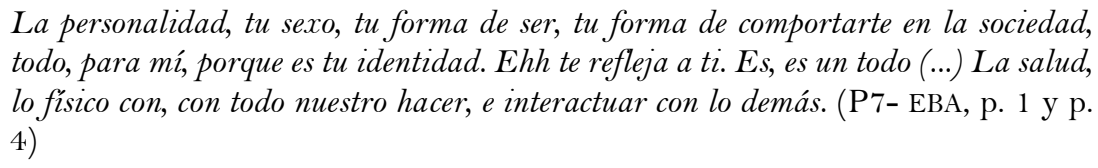

\section{e) La identidad sexual se construye y está en desarrollo permanente}

Un quinto conocimiento declarativo de identidad sexual que emergió de la mayoría de los profesores y profesoras de EBA, se refiere a la identidad sexual como una construcción y desarrollo permanente entendido según Robles (2008). Los profesores definen la identidad sexual como una construcción y desarrollo permanente.

No, no es estático, es algo que va evolucionando, o sea no debería ser, pero está siendo, el tiempo lo va dando, todas las cosas van cambiando, todo tiene su cambio (...) la 
identidad sexual se va dando con el tiempo, se va reafirmando con el tiempo. (P3EBA, p.1)

\section{f) Identidad sexual: una influencia de la familia}

Un sexto conocimiento declarativo que emerge, es que la familia influye en la construcción de la identidad sexual. Es decir, que mediante la crianza, como también mediante los referentes, el niño y la niña van aprendiendo conductas de sus padres. La crianza y los modelos se generan, como plantea Eccles (1999) y Crawford y Bodine (1997, en Mangrulkar, Whitman y Posner, 2001):

Aqui hay niñitos que de repente, cosas tan simples, como que se caen (inspira profundamente) y están ahí (gesto de aguantarse las ganas de llorar), pero que no lloran, porque según ellos, el papá les dice que los hombres no lloran. (P2- EBA, p. 2)

Sintesis

En los discursos de los y las entrevistados/as se observa que la mayoría define la identidad sexual desde un paradigma esencialista, a partir del sexo biológico, en el cual se desconoce la construcción social, cultural e histórica de la misma. No obstante, solo una persona lo define como un concepto multidimensonal, en donde involucra una construcción permanente y continua, observando que tal diferencia paradigmática entre los y las entrevistadas tiende a la concepción esencialista más que a una comprensión social y cultural.

\subsection{Conocimientos actitudinales: Profesoras y profesores influyen en la construcción de IS}

Las categorías que emergieron en relación a los conocimientos actitudinales son tres: A) influye profesor, B) según el tipo de establecimiento y C) a través de la escuela.

Tabla 4. Conocimientos actitudinales. Profesoras y profesores influyen en la construcción de la IS

\begin{tabular}{ll}
\hline \multicolumn{1}{c}{ CATEGORÍAS EMERGENTES } & \multicolumn{1}{c}{ SubCATEGORía EMERGENTE } \\
\hline & G.1 A través de su saber pedagógico. \\
A. Influye profesor & G.2 A través de sus características personales. \\
& G.3 Como profesor de educación básica. \\
& G.4 Como profesor de educación parvularia. \\
\hline \multirow{2}{*}{ B. Según el tipo de establecimiento } & H.1 Municipal. \\
\hline \multirow{2}{*}{ C. A través de la escuela } & H.2 Religioso. \\
\hline
\end{tabular}

Fuente: Elaboración propia.

a) Identidad sexual: influencia como profesor o profesora.

Un primer conocimiento actitudinal sobre identidad sexual que tiene un grupo de profesores y profesoras, refiere a que influye profesor o profesora, de acuerdo a lo planteado por De Tezanos (2007). Un ejemplo de esto:

Todo el tiempo, todo el tiempo, sobre todo los profesores de básica, más todavía primer ciclo, más aún todavía los niños están absolutamente llanos a... y porque no decirles vulnerables todavía, todavía están dispuestos abiertos a lo que el profesor les entrega, por lo tanto uno al tratar de formar no puede formar específicamente los contenidos conceptuales, uno está formando todo el tiempo y eso es esencial, la identidad como yo trato la diferencias entre las niñas y los niños si los pongo a la par, si introduzco en 
ellos que ciertas capacidades las tienen sólo los niños las tienen sólo las niñas, entonces estoy enseñando todo el tiempo en su formación. (P5- EBA, p. 1)

b) Influencia según el tipo de establecimiento.

Un segundo conocimiento actitudinal sobre identidad sexual que tienen los profesores y profesoras de EPA y EBA, refiere a que el tipo de establecimiento, sea municipal o religioso, influye en la construcción de ésta. Un ejemplo es:

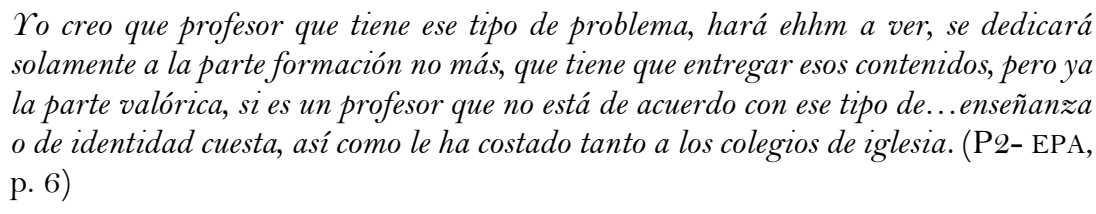
solamente a la parte formación no más, que tiene que entregar esos contenidos, pero ya la parte valórica, si es un profesor que no está de acuerdo con ese tipo de...enseñanza o de identidad cuesta, así como le ha costado tanto a los colegios de iglesia. (P2- EPA, p. 6)

c) Influencia a través de la escuela.

Un tercer conocimiento actitudinal de identidad sexual que tienen profesores y profesoras de EPA y EBA refiere a la escuela. en este estudio de investigación, escuela es entendida como un espacio de obstáculos y facilitadores para la construcción de la identidad sexual en estudiantes. y en una de las subcategorías de la escuela fueron los planes y programas de estudio, los cuales son entendidos como documentos guías que prescriben las finalidades, contenidos y acciones que son necesarios para llevar a cabo por parte el profesor o profesora, y sus alumnos y alumnas para desarrollar un currículum (Casarini, 1999).

To siempre trabajo con ellos un programa que parte del autoconcepto para llegar a fortalecer el autoestima, entonces en el autoconcepto tengo que identificarme yo con mis diferencias por supuesto. ( $\mathrm{P} 5-\mathrm{EBA}$, p. 2)

Sintesis

En relación a los hallazgos actitudinales, los profesores y las profesoras concuerdan en su mayoría que influyen en la construcción de la identidad sexual, así como también lo haría la escuela, pero se establecen distinciones en torno al tipo de establecimiento y proyecto educativo de los mismos.

\subsection{Conocimientos procedimentales sobre identidad sexual de profesores y profesoras}

Las categorías que emergieron en relación al conocimiento procedimental sobre identidad sexual son tres: A) El contenido, B) La metodología y C) La autoformación

\section{a) Trabajo de la identidad sexual a través del contenido}

Un primer conocimiento procedimental de identidad sexual que tienen profesores y profesoras de EPA y EBA refiere a que trabajan la identidad sexual a través del contenido. Tanto en las bases curriculares de EPA y los planes y programas de EBA se entiende el concepto de contenido, como "aquellos aprendizajes esperados para el nivel educativo y la edad de las y los estudiantes, que permiten desarrollar el pensamiento, permitiéndoles ejercitar su iniciativa y su capacidad de emprender proyectos" (MINEDUC, 2012). Los contenidos que los profesores y las profesoras trabajan son: lo biológico, la afectividad y las emociones, lo psicológico, los valores; las asignaturas y las unidades temáticas, por ejemplo: 
Lo biológico:

Ehh que el niño diferencia bien los genitales, que sepa que el nombre de los genitales del hombre son testículos, pene, las mujeres, la vulva, la vagina. (P2- EPA, p.1)

Los valores:

Nosotros tenemos alumnos que son repitentes ha pasado y en este curso pasó también, hago un trabajo en que el niño trate de integrarse al curso, en sus nuevos compañeros, entonces todos tienen que aceptarlo está prohibido reírse de él o de ella porque era de otro curso porque no aprendió, le acostó aprender y como tal ese niño tiene que ser capaz de respetar al otro porque él tienen derecho a expresarse por ejemplo ehh no acepto los sobre nombres en este caso no sé otros colegas pero si en mi sala de clases nos tratamos como personas que somos porque valemos todos tienen derecho a expresarse, tienen opinión y si yo interrumpo a otro no lo dejo expresarse, estoy en contra de su derecho a expresión. (P8- EBA, p. 2)

Tabla 5. Conocimientos procedimentales de profesores y profesoras, sobre identidad sexual

\begin{tabular}{ll}
\hline \multicolumn{1}{c}{ CATEGORÍAS EMERGENTES } & \multicolumn{1}{c}{ SUBCATEGORÍAS EMERGENTES } \\
\hline & J.1 Biológico \\
& J.2 Afectividad y emociones \\
A. Contenido & J.3 Psicológico \\
& J.4 Valores \\
& J.5 Asignaturas \\
& J.6 Unidades temáticas \\
\hline & K.1 Trabajo colaborativo \\
& K.2 Método inductivo \\
B. Metodología & K.3 Transversalmente \\
& K.4 Identidad de género \\
& K.5 Familia \\
\hline C. Autoformación & L.1 Identidad sexual \\
\hline
\end{tabular}

Fuente: Elaboración propia.

b) Trabajo de la identidad sexual a través de la metodología

Un segundo conocimiento procedimental sobre identidad sexual que tienen los profesores y las profesoras es la metodología, en la cual es posible evidenciar las siguientes subcategorías: trabajo colaborativo, método inductivo, transversal, identidad de género, sexo y familia.

Trabajo colaborativo:

Tengo "Yo, mi familia y mi comunidad" por ejemplo trabajo con el profesor Juan Luis (Educador Diferencial del establecimiento) y alli estamos trabajando el cuerpo humano, la sexualidad del niño, y lo abordamos juntos. (P3- EPA, p. 2)

\section{c) Trabajo de la identidad sexual a través de la autoformación}

Un tercer conocimiento procedimental de identidad sexual que tienen los profesores y las profesoras refiere a la autoformación como medio para trabajar la identidad sexual. Un ejemplo de esto es:

Hay cosas que yo no entiendo que no sé y las estudio y pregunto y se las pregunto a personas como ustedes los diferenciales que forman un rol importante, o está el psicólogo que la sexualidad mira no es así, no todo lo sé, lo voy aprendiendo. (P3EPA, p. 3) 


\section{Sintesis}

Los profesores y las profesoras concuerdan en el trabajo de la identidad sexual a través de los contenidos y la metodología, es solo un profesor que plantea la autoformación como conocimiento procedimental. Este aspecto da cuenta que en su mayoría se ciñen completamente a las directrices de los planes curriculares, y en solo un caso existiría cuestionamientos críticos y procesos de autoformación en las temáticas planteadas.

\section{Discusión}

A partir de los hallazgos es posible afirmar que tanto profesores y profesoras de EBA y EPA, consideran que existe un factor biológico que compone la identidad sexual. Sin embargo, la mitad de las profesoras de EBA establecieron que el sexo biológico y el género se configuran en una relación causal inalienable, es decir, si una persona tiene un sexo definido como hombre, éste necesariamente debe ser masculino. Este último elemento nos remite al concepto de género discutido en el marco referencial, el cual se relaciona al sentirse hombre o sentirse mujer, sin embargo, no es una causalidad del sexo biológico, como bien es sabido a partir de la experiencia y conocimiento de niños y niñas transexuales.

Por otra parte, dos profesoras de EBA, y la mayoría de las educadoras de párvulos afirmaron que el género y la orientación sexual vienen determinados por el sexo biológico de la persona, comprendiendo estos componentes de la identidad sexual como unificados, inalienables y estáticos, por lo que no existirían factores contextuales que lo afecten, cambien y/o modifiquen. Sin embargo, es sabido que la identidad sexual es dinámica (Conejero y Almonte, 2009). En este sentido el profesorado entrevistado, entiende que la orientación sexual se compone solo por una orientación heterosexual, por lo que la homosexualidad no se configuraría como otro tipo de orientación sexual, por lo tanto, tampoco como un componente de la identidad. Esta comprensión se condice con elementos de injusticia de reconocimiento tal como lo plantea Fraser (2008); estableciéndose como un dispositivo de poder y dominación (Friend, 1993 en Cornejo, 2010) del mundo heterosexual y adulto por sobre el homosexual y el de la niñez. Asimismo, el sexo y el género pueden entenderse como una construcción del cuerpo a partir de actos ritualizados y dogmáticos que corresponderían a lo que Butler (1993 y 2003, en Duque, 2010) denomina Matriz Heterosexual, en donde es posible de observar prácticas de crianza, juegos, modos de vestir y enseñanzas, que perpetúan condiciones únicas y genéricas de estos elementos.

Pese a que existen confusiones en torno a los conceptos relacionados con la identidad sexual y sus componentes, existe consenso en que, todos y todas tienen derecho a una identidad (UNESCO, 1990), y que esta se compone, entre otras, por la sexualidad y afectividad. Algunos de los profesores y las profesoras de EPA y EBA, refieren la identidad como algo esencial de la persona, la cual viene definida por un factor genético. En cambio otros, afirman que las identidades se desarrollan y cambian a través del tiempo. Los primeros sustentan sus conocimientos en un enfoque esencialista, el cual no se condice con un enfoque de desarrollo y construcción identitario como el definido por Erikson (1963, en Robles, 2008) y Almonte (2007). El segundo grupo, concuerda con los planteamientos de Erikson, ya que identifican que las personas mediante las experiencias van construyendo su identidad, partiendo desde la niñez temprana. Asimismo, Castellano 
(2004, en Duque, 2010) agrega que no tenemos una identidad fija e inherente, sino que somos capaces de utilizar y desarrollar identidades para distintos momentos, con ciertos elementos más estables, pero que fluctúan durante toda la vida.

Los resultados del estudio, permiten inferir que las educadoras de párvulos son el grupo de docentes que tienen mayor conocimiento sobre el concepto de identidad sexual. Una hipótesis posible de manejar es que esto se debe a que trabajan considerando las Bases Curriculares para el nivel que explicitan el principio de singularidad de las y los párvulos y que el núcleo curricular refiere a la identidad y autonomía de ellos y ellas. Sin embargo, los profesores y las profesoras de EBA, si bien trabajan aspectos biológicos, la finalidad no radica en el desarrollo de identidad sexual, sino en asumir la responsabilidad por la salud y el cuidado de su cuerpo (MINEDUC, 2012). Por lo tanto, los conocimientos declarativos de profesoras y profesores, tienen directa relación con los contenidos planteados en el currículo regular de cada nivel educativo.

La mayoría de los profesores y profesoras de EPA y EBA, definen la identidad sexual como un concepto multidimensional que integra aspectos culturales, afectivoemocionales y la multidimensionalidad del ser. No obstante, ellos y ellas definen esta multidimensionalidad a través del "sentir" y del "relacionarse con otros" y no fundamentando teóricamente sus conocimientos.

En relación a los conocimientos actitudinales que emergieron del estudio, es posible afirmar que algunas de las educadoras de Párvulos indican que sus características personales influyen, en las interacciones con los párvulos (Vigotsky, 1956, en Castorina, Ferreiro, Kohl y Lerne, 1996). Asimismo, abordan la identidad sexual desde una perspectiva biologicista, haciendo alusión a aspectos físicos más que a la afectividad, como un elemento de la construcción de la identidad sexual.

También los profesores y las profesoras afirman que trabajan la identidad sexual desde diversos contenidos, puesto que consideran que no existen planes y programas que aborden los componentes de ésta, lo que genera que sean trabajados a partir de sus propias convicciones. Por último, una educadora de párvulos, y una profesora de EBA, mencionaron que dependiendo del tipo de establecimiento, se puede aportar u obstaculizar la formación en torno a la construcción de la identidad sexual, en tanto, al poseer libertad de enseñanza, los establecimientos tienen la opción de elegir la información que ellos consideren pertinente y necesaria de abordar en temáticas de sexualidad e identidad.

Los resultados del estudio también indican que los algunos profesores de educación básica sitúan los conocimientos procedimentales sobre la identidad sexual desde una labor pedagógica. En éste sentido el profesorado influye en la construcción de la IS desde la enseñanza de los contenidos que entregan a sus alumnos y alumnas. En el caso de las Educadoras de Párvulos influyen desde los contenidos referidos al reconocimiento del cuerpo, su sexo y género. Las profesoras de primer y segundo año de EBA, influyen desde la enseñanza de la identificación de las partes del cuerpo, pero dando énfasis en la funcionalidad, el autocuidado y la vida saludable, tal como se menciona en las Bases Curriculares de ciencias naturales, y educación física y salud (MINEDUC, 2012). Dentro de ese grupo, la mitad de los profesores y las profesoras de EBA, abordan la identidad sexual desde una perspectiva multidimensional, relacionando los aspectos biológicos, emocionales y psicológicos. De ello, se puede inferir que no tienen interiorizado un fundamento teórico que les permita abordar estas temáticas de manera consciente, tal como lo afirma García, Sala, Rodríguez y Sabuco (2013). Sin embargo, no todos/as los 
profesores y las profesoras tienen una mirada constructivista, dado que abordan unidimensionalmente la identidad sexual, es decir, sólo desde lo biológico y desde un enfoque esencialista.

Con respecto a las metodologías que utilizan profesores y profesoras para influir en la construcción de la identidad sexual, se encuentran métodos inclusivos como el trabajo colaborativo que plantea Sapon-Shavin (2008). Sin embargo, se cuestiona la efectividad de estas metodologías, debido a que no tienen fundamentos teóricos que sustenten sus métodos, los cuales además se restringen a un enfoque esencialista (García et al., 2013).

Asimismo, los profesores y profesoras declaran que es necesaria una instancia de educación formal, en relación a la temática de identidad sexual, ya que en la actualidad los conocimientos sobre ésta y sus componentes dependen de la autoformación de cada docente o de los lineamientos de los establecimientos educativos. Ahora bien, tal como afirma García y colaboradores (2013), los profesores y las profesoras en la formación profesional no aprenden lineamientos sobre diversidad sexual y género, que no sean biológicos. Esto provoca que no tengan claro su quehacer pedagógico en relación a la construcción de la identidad sexual al momento de ingresar al sistema escolar, puesto que no cuentan con el saber pedagógico que le permita acompañar la formación de sus alumnos y alumnas desde un enfoque inclusivo.

Finalmente, es posible afirmar que actualmente las políticas públicas, tanto en el ámbito civil como educativo, han visibilizado problemáticas de la comunidad LGBTI, en torno a sus derechos ciudadanos como también la necesidad de desarrollar una educación que se dirija al desarrollo integral, resguardando los derechos y el enfoque de género. Pese a ello, y rescatando los avances en políticas de convivencia escolar, es posible plantear que estas políticas han sido construidas desde análisis frágiles, donde no se cuestiona la hegemonía de las sexualidades, dejando indemne el heterosexismo, los binarismos de género, el control de los cuerpos y la concepción de familia heterosexual (Echeverría y Maturana, 2015), así como también dejan fuera cuestiones de injusticia de redistribución, reconocimiento y participación como las que se asumen desde Fraser (2008) y un reconocimiento pleno de la diferencia y diversidad sexual, para la comprensión de un nuevo orden simbólico (Butler, 1993 y 2003, en Duque, 2010; Preciado, 2002). Asimismo, los intentos de introducir temáticas relacionadas al ámbito de la identidad sexual, han fortalecido la exclusión, volviendo a situar la "diversidad" como la otredad, solo alcanzando un respeto limitado hacia otro/a, pero no una valoración de la diferencia (Butler 2009, en Echeverría y Maturana, 2015) que permita afirmar que cada niño y niña, independiente de su identidad sexual, es un legítimo aprendiz (Julio, 2010, 2014).

Por lo expuesto, Chile en su actual política de convivencia escolar, la comprende como una estrategia de cambio dentro del sistema educativo, que promueve relaciones horizontales y equitativas, mediados por un lenguaje que se refiere a una multitud diversa, y no a otros diferentes (UNESCO, 2015). Sin embargo, a partir de los resultados de este estudio es posible preguntarse si estos lineamientos políticos serán capaces de configurarse como conocimientos actitudinales y procedimentales que permitan impactar el aula como un espacio de encuentros intersubjetivos (Julio, 2014) que permitan superar los desencuentros culturales en ella (Julio y otros, 2016) o más bien, seguirán perpetuándose sólo como conocimientos declarativos. Otras interrogantes que deja el estudio son si el profesorado que responde desde un paradigma socio-constructivista, tiene incorporado este enfoque en sus conocimientos procedimentales y actitudinales (Barrientos y 
Cárdenas, 2013). Finalmente también es posible preguntarse ¿La creación de leyes propicia la generación de conocimientos que trasciendan a lo declarativo? ¿Qué es lo que se necesitaría para re-pensar el saber pedagógico (desde la creación de leyes hasta el establecimiento de conocimientos procedimentales y actitudinales) para que los profesores y profesoras se transformen en facilitadores de la construcción de identidad sexual en la niñez?

\section{Referencias}

Almonte, C. (2007). Desarrollo homosexual en el adolescente. En B. Zegers y M. Larraín (Eds.), Sobre la homosexualidad (pp. 229-254). Santiago: Mediterráneo.

Bardi, A., Leyton, C., Martinez, V. y Gonzáles E. (2005). Identidad sexual: proceso de definición en la adolescencia. Reflexiones Pedagógicas, 26, 43-51.

Barrientos, J. y Cárdenas, M. (2013). Homofobia y calidad de vida de gay y lesbianas: una mirada psicosocial. Psykhe, 22(1), 3-14. doi:10.7764/psykhe.22.1.553

Becerra, M. y Melo, O. (2006). Identidad sexual y desarrollo de la personalidad. Madrid: Asociación Mundial de Educadores Infantiles.

Brooker, L. y Woodhead, M. (2008). El desarrollo de identidades positivas: la diversidad y la primera infancia. Londres: The Open University.

Casarini, M. (1999). Teoría y diseño curricular. México: Trillas.

Cáceres, P. (2003). Análisis cualitativo: una alternativa metodológica alcanzable. Psicoperspectivas, 2, 53-82.

Castorina, J., Ferreiro, E., Kohl, M. y Lerner, D. (1996). Piaget-Vigotsky: contribuciones para replantear el debate. Barcelona: Paidós.

Conejero, J. y Almonte, C. (2009). Desarrollo de la orientación sexual en adolescentes de 16 a 18 años de ambos sexos de Santiago de Chile. Revista Chilena Neuro-Psiquiatrica, 47, 201-208. doi:10.4067/s0717-92272009000300004

Cornejo, J. (2010). Jóvenes en la encrucijada. Última Década, 32(18), 173-189. doi:10.4067/s071822362010000100010

Debís, J., Fuentes, J. y Sparkes, A. (2005). ¿Qué permanece oculto del curriculum oculto? Las sexualidades de género y sexualidades en la educación física. Revista Iberoamericana de Educación, 21(18), 73-90.

Delors, J., Mufti, I. A., Amagi, I., Carneiro, R., Chung, F., Geremek, B. y Nanzhao, Z. (1996). Informe de la UNESCO de la comisión internacional sobre educación para el siglo XXI. La educación encierra un tesoro. París: UNESCO.

Doménech, F. (1999). Proceso de enseñanza/aprendizaje universitario. Castellón: Ediciones de la Universitat Jaume I.

Duque, C. (2010). Judith Butler y la teoría de la performatividad de género. Revista de Educación y Pensamiento, 17, 85-95.

Echeverría, G. y Maturana, J. M. (2015). Análisis crítico del discurso de políticas públicas en diversidad sexual en Chile. Universitas Psychologica, 14(4), 1485-1498.

Fraser, N. (2008). La justicia social en era política de identidad: redistribución, reconocimiento y participación. Revista de Trabajo, 4(6), 83-99. 
García, R., Sala, A., Rodriguez, E. y Sabuco, A. (2013). Formación inicial del profesorado sobre género y coeducación: impactos metacognitivos de la inclusión curricular transversal sobre sexismo y homofobia. Profesorado. Revista de Currículum y Formación del Profesorado, 17(1), 270-284.

Grinell, R. (1997). Social work research ङ evaluation: quantitative and qualitative approaches. Itaca, IL: Peacock Publishers.

Hemingway, J. (2008). Speaking the unspeakable in forbidden places: addressing lesbian, gay, bisexual and transgender equality in the primary school. Sex Education: Sexuality Society and Learning, 8(3), 315-328. doi:10.1080/14681810802218395

Hernández, R., Fernández, C. y Baptista, P. (2003). Metodología de la investigación. México: McGraw-Hill.

Julio, C. (2010). Legítimos aprendices: recuperando al sujeto en el proceso educativo. Revista Diversia, 2, 19-43.

Julio, C. (2014) Dilema identitario en el primer ciclo de educación básica municipal: un obstáculo en la relación pedagógica que incide en la posibilidad de concluir trayectorias de aprendizaje escolar, (Tesis doctoral). Universidad Academia de Humanismo Cristiano. Chile.

Julio-Maturana, C., Conejeros-Solar, L., Rojas, C., Mohammad, M., Rubí, Y. y Cortés, Y. (2016). Desencuentro cultural en el aula: Una barrera al aprendizaje de niños y niñas en situación de pobreza. Revista Mexicana de Investigación Educativa, 21(68), 71-94.

Lincoln, Y. y Guba, E. (1985). Naturalistic inquiry. Londres: Sage.

Mangrulkar, L., Whitman, C. y Posner, M. (2001). Enfoque de habilidades para la vida para un desarrollo saludable de niños y adolescentes. Washington, DC: Organización Panamericana de la Salud.

Osses, S., Sánchez, I. y Ibáñez, F. (2006). Investigación cualitativa en educación. Hacia la generación de teoría a través del proceso analítico. Estudios Pedagógicos, 32(1), 119-133. doi:10.4067/s0718-07052006000100007

Odreman, N. (1996). Proyecto educativo de educación básica, reto compromiso y transformación. Educación, 179, 13-43.

Polaino-Lorente, A. (2007). El desarrollo de la identidad sexual en los varones: líneas de actuación en el tercer milenio. Revista Española de Pedagogía, 238, 397-432.

Pozo, J. y Monereo, C. (1999). Aprendizaje estratégico. Santillana: Madrid.

Preciado, B. (2002). Manifiesto contra-sexual. Práticas subversivas de identidad sexual. Madrid: Opera Prima.

Prengel, A. (2011). Ziele einer geschlechterdemokratischen. Pädagogik im inklusiven Kindergarten. Zeitschrift für Inklusion, 5(1), 1-24.

Prieto, M. (2004). La construcción de la identidad profesional docente: un desafío permanente. Revista Enfoques Educacionales, 6(1), 29-49.

Tezanos, A. (1983). Notas para una reflexión crítica sobre la pedagogía. En G. Hoyos (Ed.), El sujeto como objeto en las ciencias sociales (pp. 314-334). Bogotá: CINEP

Tezanos, A. (2007). Formación de profesores: una reflexión y una propuesta. Revista Pensamiento Educativo, 41(2), 57-75.

Robles, B. (2008). La infancia y la niñez en el sentido de identidad. Comentarios en torno a las etapas de la vida de Erik Erikson. Revista Mexicana de Pediatría, 75, 29-34. 
Romero, I. (2010). Acompañar los primeros pasos de los docentes. Santiago de Chile: Santillana.

Sapon-Shevin, M. (2008). Learning in an inclusive community. Educational Leadership, 66(1), 4953.

UNESCO. (1990). Declaración mundial sobre educación para todos: satisfacción de las necesidades básicas de aprendizaje. Jomtien: WCEFA.

UNESCO. (2015). La violencia homofóbica y transfóbica en el ámbito escolar: hacia centros educativos inclusivos y seguros en América Latina. Recuperado de http://www.convivenciaescolar.cl/

Vargas, I. (2012). La entrevista en la investigación cualitativa: nuevas tendencias y retos. Revista Calidad en la Educación Superior, 3(1), 119-139.

Woolfolk, A. (2006). Psicología educativa. Madrid: Pearson

\section{Breve CV de los autores}

\section{Cristina Julio Maturana}

Consultora Independiente y Docente Universidad de Playa Ancha de Ciencias de la Educación (UPLACED). Email cristina.julio@upla.cl

\section{Anna Kaeuffer}

Licenciada. Profesora de Educación Diferencial con mención en Trastornos de Aprendizaje Específico de la Pontificia Universidad Católica de Valparaíso. Email: akaeuffer@gmail.com

\section{Christopher Riquelme Salinas}

Licenciado. Profesor de Educación Diferencial con mención en Trastornos del Aprendizaje Específico de la Pontificia Universidad Católica de Valparaíso. Email: chris.riquleme.salinas@gmail.com

\section{María Paz Silva Erices}

Licenciada. Profesora de Educación Diferencial con mención en Trastornos del Aprendizaje Específico de la Pontificia Universidad Católica de Valparaíso. Email: mariapaz.silvaerices@gmail.com

\section{María Roswitha Osorio Hodges}

Licenciada.. Profesora de Educación Diferencial con mención en Discapacidad Intelectual de la Pontificia Universidad Católica de Valparaíso. Email: roswithaosoriohodges@gmail.com 


\section{Natalie Torres Estay}

Licenciada. Profesora de Educación Diferencial con mención en Trastornos de Aprendizaje Específico de la Pontificia Universidad Católica de Valparaíso. Email: natalie.torres.estay@gmail.com 